\title{
Sound Radiation Mitigation of Geometrically Nonlinear Plates Subjected to Subsonic Airflow
}

\author{
Hamed Norouzi (iD) ${ }^{1}$ and Davood Younesian (iD) ${ }^{2}$ \\ ${ }^{1}$ Department of Railway Engineering and Transportation Planning, Faculty of Civil Engineering and Transportation, \\ University of Isfahan, Isfahan 81746-73441, Iran \\ ${ }^{2}$ Center of Excellence in Railway Transportation, School of Railway Engineering, Iran University of Science and Technology, \\ Tehran 16846-13114, Iran \\ Correspondence should be addressed to Hamed Norouzi; h.norouzi@eng.ui.ac.ir
}

Received 6 May 2021; Revised 6 June 2021; Accepted 12 July 2021; Published 20 July 2021

Academic Editor: Francesc Pozo

Copyright (c) 2021 Hamed Norouzi and Davood Younesian. This is an open access article distributed under the Creative Commons Attribution License, which permits unrestricted use, distribution, and reproduction in any medium, provided the original work is properly cited.

\begin{abstract}
This paper gives a theoretical analysis to obtain and reduce the acoustic pressure generated from plates with geometric nonlinearities subjected to subsonic airflow and external excitation. von-Kármán assumptions are applied considering the nonlinear terms in strain-displacement relations. Airflow passing through the plate is considered as an incompressible, irrotational, and inviscid flow. Galerkin's method is employed to acquire the governing equations of time-dependent coefficients. Multiple Time Scale Method (MTSM) is then used to obtain the response of the plate. Suppressing undesirable vibration is carried out using an optimal tuned mass damper (TMD) system and an analytical solution is proposed based on Laplace transform and Adomian Decomposition Method (ADM). The acoustic pressure received from the plate is calculated by solving the Rayleigh integral using Boundary Element Method (BEM). A parametric study is carried out, and the effects of the flow speed, the aspect ratio, the thickness of the plate, the material of the plate, the forcing frequency, and the effectiveness of the designed TMD system on the sound pressure are examined. According to the results, using the TMD system reduces the amplitudes of the plate vibrations and, consequently, reduces the acoustic pressure around the vibrating plate. In this study, the passive control strategy leads to a significant decrease in the sound pressure level (about 35\%) in some airflow speeds. Results show good efficiency in the control strategy. It is also found that the acoustic pressure generated from steel plates is significantly larger than that generated from aluminum plates. Moreover, increasing the aspect ratio and the plate thickness reduces the acoustic pressure. On the other hand, the external excitations with lower frequencies and the airflows with higher densities can generate lower sound pressures around the plate.
\end{abstract}

\section{Introduction}

Plates are widely used in engineering applications, especially in automobile structures and high-speed trains (HST) [1]. Such plates are commonly considered elastic or viscoelastic. It can be noted that the viscoelasticity effects may be examined in other mechanical structures such as disks, beams, or gyroscopes [2]. Anyway, the plates used in high-speed trains are typically subjected to a subsonic airflow. Reducing the noise generation is unavoidable for passenger comfort in the HST. So, analyzing the sound radiation from the plates in airflows and reducing the levels of this noise seem to be necessary. Moreover, there is a paucity of research on the analytical noise control of these plates. In the last decades, many types of researches have been established about vibrations and sound radiation from the linear and nonlinear plates. For example, Sorokin [3] examined the vibrations of and sound radiation from sandwich plates in heavy fluid loading conditions using a semianalytical approach. Chong et al. [4] performed a theoretical analysis of the vibration and the sound radiation generated by plates excited by piezoelectric actuators. Forced vibration of rectangular orthotropic plates under moving loads was studied using Rayleigh integral technique by Au and Wang [5]. Akamatsu et al. [6] 
analyzed sound radiation from baffled finite elastic plates that are subjected to impulses occurring at random points on the surface of the plate. Denli and Sun [7] presented an optimization study of sandwich structures with cellular cores for minimum noise radiation in a wide frequency band, subject to the constraints on the fundamental frequency and weight. Jeyaraj et al. [8] numerically studied the vibration and acoustic response characteristics of an isotropic rectangular plate in a thermal environment. They found that the overall sound radiation of the plate increases with an increase in temperature for all boundary conditions. An analytical model framework able to accurately predict the flowinduced noise in the interior of a transport vehicle cabin was presented by Rocha et al. [9]. An analytical approach was formulated by Xin and $\mathrm{Lu}$ [10] to account for the effects of mean flow on sound transmission across a simply supported rectangular aeroelastic panel. Smith and Iglesias [11] examined vibration and noise radiation from a panel excited by a turbulent flow. Rocha [12] investigated the vibration and sound radiation by panels exited by turbulent flow and by random noise. Sucheendran et al. [13] proposed an analytical solution for the fully coupled structural-acoustic response of a thin elastic plate mounted in a rectangular duct. Akgöz and Civalek [14] developed a new nonclassical sinusoidal plate model based on modified strain gradient theory. Yang et al. [15] presented an analytical study for sound radiation of functionally graded materials (FGM) plate based on the three-dimensional theory of elasticity. Shakeri and Younesian [16] studied the acoustic radiation in the transient and steady-state modes for a clamped-free annular plate with a tuned mass damper (TMD) device. Daneshjou et al. [17] proposed an exact solution of three-dimensional elasticity for sound transmission loss through an FG cylinder in presence of subsonic external flow. The vibroacoustic modeling and analysis of the sandwich plates with metalceramic functionally graded (FG) core are presented by Chandra et al. [18]. Song et al. [19] proposed a method of panel flutter suppression and elimination for aeroelastic structures in supersonic airflow. Minimization of sound radiation in fully coupled structural-acoustic systems was performed by Zhao et al. [20] using FEM-BEM based topology optimization. Chauvin et al. [21] examined a clamped thin membrane made in Latex in order to develop a nonlinear vibroacoustic absorber in the acoustic domain. The vibration and sound radiation of porous functionally graded material (FGM) plates with a temperature gradient along the thickness were studied by Zhou et al. [22]. Jalaei and Civalek [23] examined the dynamic instability of viscoelastic porous functionally graded (FG) nanobeam embedded on viscoPasternak medium subjected to an axially oscillating loading as well as a magnetic field. Qin et al. [24] studied sound radiation from plates with elastic boundary conditions embedded in an infinite perforated rigid baffle. Additionally, Huo et al. [25] proposed the optimized dynamic vibration absorbers for suppressing sound radiation of plate structures. Recently, the authors of [26] obtained transient and steady-state acoustic pressure of viscoelastic resonant plates using Adomian Decomposition Method (ADM) and Durbin's numerical Laplace transform inversion technique.

A survey of the literature reveals that not many studies have been performed in the area of vibration and acoustic responses of plate structures subjected to a fluid field. Additionally, there is a lack of analytical modeling in three dimensions for the vibrating plates and the fluid flow pressure in order to calculate and reduce the sound levels generated by the plates. In the present study, this gap in the literature has been covered and a new solution based on Laplace transform and Adomian Decomposition Method has been proposed to represent the response of the plateabsorber system analytically.

\section{Mathematical Modeling}

2.1. Governing Equations and General Solution. Schematic representation of a simply supported plate subjected to a subsonic airflow has been illustrated in Figure 1. An external excitation of the form $\tilde{f}(x, y, t)=\widetilde{f}_{0} \sin (\pi x)$ a) $\sin (\pi x / a) \cos \psi t$ is simultaneously acting on the surface of the plate. The dimensions of the plate have been demonstrated in Figure 1. Moreover, the mass density and the speed of the airflow are $\rho_{\infty}$ and $U_{\infty}$, respectively.

Neglecting the viscoelasticity effect, the governing equation of motion of the plate can be expressed as $[1,26]$

$$
\begin{aligned}
& D \nabla^{4} w+c h \dot{w}+\rho h \ddot{w}-\tilde{f}-P_{a}-\left[\left(\frac{E h}{2 a\left(1-v^{2}\right)} \int_{0}^{a}\left(\left(\frac{\partial w}{\partial x}\right)^{2}+v\left(\frac{\partial w}{\partial y}\right)^{2}\right) \mathrm{d} x\right)\left(\frac{\partial^{2} w}{\partial x^{2}}\right)\right. \\
& +\left(\frac{E h}{2 b\left(1-v^{2}\right)} \int_{0}^{b}\left(\left(\frac{\partial w}{\partial y}\right)^{2}+v\left(\frac{\partial w}{\partial x}\right)^{2}\right) \mathrm{d} y\right)\left(\frac{\partial^{2} w}{\partial y^{2}}\right) \\
& +\frac{E h}{(1+v)} \frac{\partial w}{\partial x} \frac{\partial w}{\partial y} \frac{\partial^{2} w}{\partial x \partial y}=0,
\end{aligned}
$$

where $a, b, w, D, E, \rho$, and $v$ are length, width, deflection, rigidity, modulus of elasticity, mass density, and Poisson's ratio of the plate, respectively, and one can define
$D=\left(E h^{3} /\left(12\left(1-v^{2}\right)\right)\right)$ and $\nabla^{4}=\left(\left(\partial^{2} / \partial x^{2}\right)+\left(\partial^{2} / \partial y^{2}\right)\right)^{2} \cdot P_{a}$ is also the pressure of the airflow on the surface of the plate. The authors of [1] reported that the response of equation (1) 


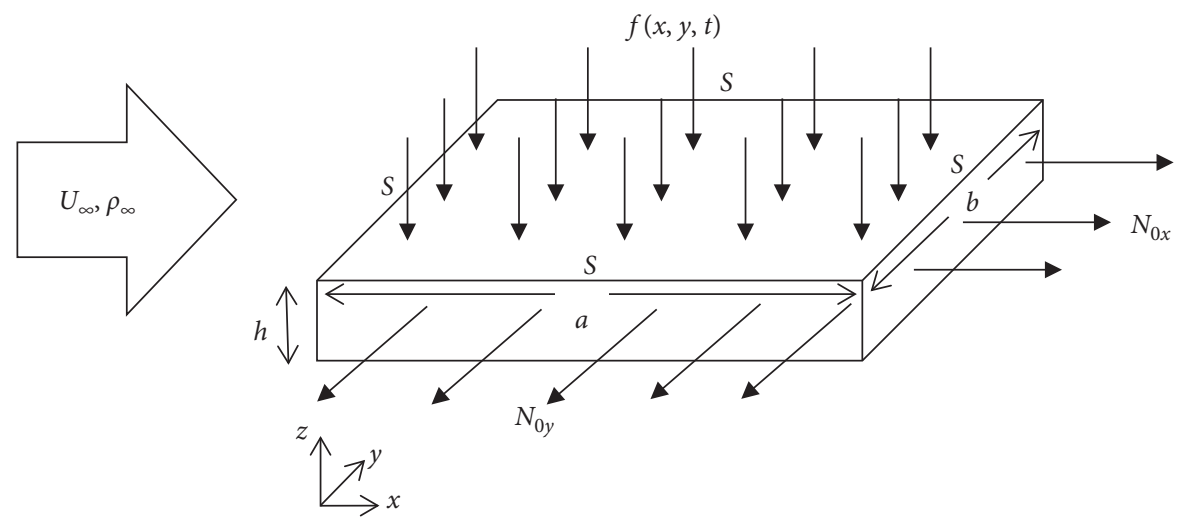

FIGURE 1: Schematic representation of a simply supported plate subjected to a subsonic airflow and external load.

could be represented by the main mode consideration; that is, $w(x, y, t)=\sin (\pi x / a) \sin (\pi y / b) q(t)$. Moreover, one can ignore the compressibility effect of the flow and clarify $P_{a}$ as [26]

$$
P_{a}(x, y, t)=\frac{\rho_{\infty} U_{\infty}^{2}}{a \sqrt{(\pi / a)^{2}+(\pi / b)^{2}}} \sin \frac{\pi y}{b}\left[\left(\frac{a}{U_{\infty}^{2}} \ddot{q}-\frac{\pi^{2}}{a} q\right) \sin \frac{\pi x}{a}+2 \frac{\pi}{U_{\infty}} \dot{q} \cos \frac{\pi x}{a}\right] .
$$

Substituting equation (2) into (1) and multiplying both sides of it by $\sin (\pi x / a) \sin (\pi y / b)$ and then performing integral from 0 to $a$ for $x$ and from 0 to $b$ for $y$ leads to obtaining governing equation of $q(t)$ as

$$
M \ddot{q}+\widetilde{C} \dot{q}+K q+\widetilde{N} q^{3}=\widetilde{F}(t),
$$

where

$$
\begin{aligned}
M & =\rho h-\frac{\rho_{\infty}}{\omega}, \\
\widetilde{C} & =c h, \\
K & =D \omega^{4}+\frac{\rho_{\infty} \pi^{2} U_{\infty}^{2}}{a^{2} \omega}, \\
\widetilde{N} & =\frac{E h}{16\left(1-v^{2}\right)}\left(3\left(\frac{\pi}{a}\right)^{4}+3\left(\frac{\pi}{b}\right)^{4}+(1+v)\left(\frac{\pi}{a}\right)^{2}\left(\frac{\pi}{b}\right)^{2}\right),
\end{aligned}
$$

$\widetilde{F}(t)=\widetilde{f}_{0} \cos \psi t$, and $\omega=\sqrt{(\pi / a)^{2}+(\pi / b)^{2}}$. Assuming $\widetilde{C}, \widetilde{N}$, and $\widetilde{F}(t)$ from small orders (say $\varepsilon$ ), one can rewrite equation (3) in the new form as

$$
M \ddot{q}+\varepsilon C \dot{q}+K q+\varepsilon N q^{3}=\varepsilon F(t) .
$$

Perturbation methods give us a solution for equation (5) in the form [27]

$$
q\left(T_{0}, T_{1} ; \varepsilon\right)=q_{0}\left(T_{0}, T_{1}\right)+\varepsilon q_{1}\left(T_{0}, T_{1}\right)+O\left(\varepsilon^{2}\right) .
$$

For using the Multiple Time Scale Method, one should introduce $T_{0}=t, T_{1}=\varepsilon t, D_{0}=\left(\partial / \partial T_{0}\right)$ and $D_{1}=\left(\partial / \partial T_{1}\right)$. A new definition of time derivatives is introduced by describing the above parameters. So, one can write [27]

$$
\begin{aligned}
& \frac{\partial}{\partial t}=\frac{\partial}{\partial T_{0}} \frac{\partial T_{0}}{\partial t}+\frac{\partial}{\partial T_{1}} \frac{\partial T_{1}}{\partial t}+\cdots=D_{0}+\varepsilon D_{1}+O\left(\varepsilon^{2}\right), \\
& \frac{\partial^{2}}{\partial t^{2}}=\frac{\partial}{\partial t} \frac{\partial}{\partial t}=\left(\frac{\partial}{\partial t}\right)^{2}=\left(D_{0}+\varepsilon D_{1}+O\left(\varepsilon^{2}\right)\right)^{2}=D_{0}^{2}+2 D_{0} D_{1} \varepsilon+O\left(\varepsilon^{2}\right) .
\end{aligned}
$$


Substituting the new time derivatives obtained in equation (7) and the solution defined in equation (6) into (5) and separating powers of $\varepsilon^{i}$ lead to [27]

$$
\begin{aligned}
& \varepsilon^{0}: M D_{0}^{2} q_{0}+K q_{0}=0 \Rightarrow q_{0}\left(T_{0}, T_{1}\right)=A\left(T_{1}\right) e^{i \sqrt{K / M} T_{0}}+c . c \\
& \varepsilon^{1}: M D_{0}^{2} q_{1}+K q_{1}=-2 M D_{0} D_{1} q_{0}-C D_{0} q_{0}-N q_{0}^{3}+f_{0} \cos \psi t \\
& =-2 i \sqrt{\frac{K}{M}} D_{1} A e^{i \sqrt{K / M} T_{0}}-C A i \sqrt{\frac{K}{M}} e^{i \sqrt{K / M} T_{0}}-N\left(A^{3} e^{3 i \sqrt{K / M} T_{0}}+3 A^{2} \bar{A} e^{i \sqrt{K / M} T_{0}}\right)+\frac{f_{0}}{2} \cos \psi t+c . c .
\end{aligned}
$$

Eliminating the secular terms gives

$$
2 i \sqrt{\frac{K}{M}} D_{1} A+i C A \sqrt{\frac{K}{M}}+N\left(3 A^{2} \bar{A}\right)=0 .
$$

Parameter $A$ is a complex number of the form

$$
A=\frac{1}{2} \alpha e^{i \beta},
$$

where $\alpha$ and $\beta$ are real numbers. Substituting equation (10) into (9) and separating real and imaginary parts construct a system of differential equations of $\alpha\left(T_{1}\right)$ and $\beta\left(T_{1}\right)$ as

$$
\left\{\begin{array}{l}
-\alpha \beta^{\prime} \sqrt{\frac{K}{M}}+\frac{3 N \alpha^{3}}{8}=0 \\
\alpha^{\prime} \sqrt{\frac{K}{M}}+\frac{\alpha C}{2} \sqrt{\frac{K}{M}}=0
\end{array}\right.
$$

The solution of equation (11) will be in the form

$$
\begin{aligned}
& \alpha(t)=\alpha_{0} e^{(-\varepsilon C t / 2)}, \\
& \beta(t)=\frac{-3 N \alpha_{0}^{2}}{8 C}\left(\frac{K}{M}\right)^{-(1 / 2)} e^{-\varepsilon C t}+\beta_{0},
\end{aligned}
$$

where $\alpha_{0}$ and $\beta_{0}$ are real constants. Obtaining $\alpha(t)$ and $\beta(t)$ leads to finding the solution of equation (8) as

$$
q(t)=\alpha(t) \cos \left(\sqrt{\frac{K}{M}} t+\beta(t)\right)+\frac{\varepsilon N(\alpha(t))^{3}}{32 K} \cos \left(3 \sqrt{\frac{K}{M}} t+3 \beta(t)\right)+\frac{\varepsilon f_{0} M}{K-M \psi^{2}} \cos \psi t
$$

and the above equation represents an analytical solution for the dynamic response of the plate.

2.2. Vibration Suppression. A suppressed plate with a tuned mass damper (TMD) system has schematically been shown in Figure 2. The mass, the stiffness, and the damping coefficient of TMD are $m_{a}, k_{a}$, and $c_{a}$, respectively, and TMD is located at the midpoint of the plate.

The governing coupled differential equations describing the new system are

$$
\begin{aligned}
& \left(D \nabla^{4} w+c h \dot{w}+\rho h \ddot{w}-\tilde{f}-P_{a}-\left[\left(N_{0 x}+\frac{E h}{2 a\left(1-v^{2}\right)} \int_{0}^{a}\left(\left(\frac{\partial w}{\partial x}\right)^{2}+v\left(\frac{\partial w}{\partial y}\right)^{2}\right) \mathrm{d} x\right)\left(\frac{\partial^{2} w}{\partial x^{2}}\right)+\left(N_{0 y}+\frac{E h}{2 a\left(1-v^{2}\right)} \int_{0}^{b}\left(\left(\frac{\partial w}{\partial y}\right)^{2}+v\left(\frac{\partial w}{\partial x}\right)^{2}\right) \mathrm{d} y\right)\left(\frac{\partial^{2} w}{\partial y^{2}}\right)\right.\right. \\
& \left.+\frac{E h}{(1+v)} \frac{\partial w}{\partial x} \frac{\partial w}{\partial y} \frac{\partial^{2} w}{\partial x \partial y}\right]-k_{a}\left\{z_{a}(t)-w\left(\frac{a}{2}, \frac{b}{2}, t\right)\right\} \delta\left(x-\frac{a}{2}\right) \delta\left(y-\frac{b}{2}\right)-c_{a}\left\{\dot{z}_{a}(t)-\dot{w}\left(\frac{a}{2}, \frac{b}{2}, t\right)\right\} \delta\left(x-\frac{a}{2}\right) \delta\left(y-\frac{b}{2}\right)=0 \\
& \left.m_{a} \ddot{z}_{a}+k_{a}\left\{z_{a}(t)-w\left(\frac{a}{2}, \frac{b}{2}, t\right)\right\}+c_{a}\left\{\dot{z}_{a}(t)-\dot{w}\left(\frac{a}{2}, \frac{b}{2}, t\right)\right\}=0,\right)
\end{aligned}
$$




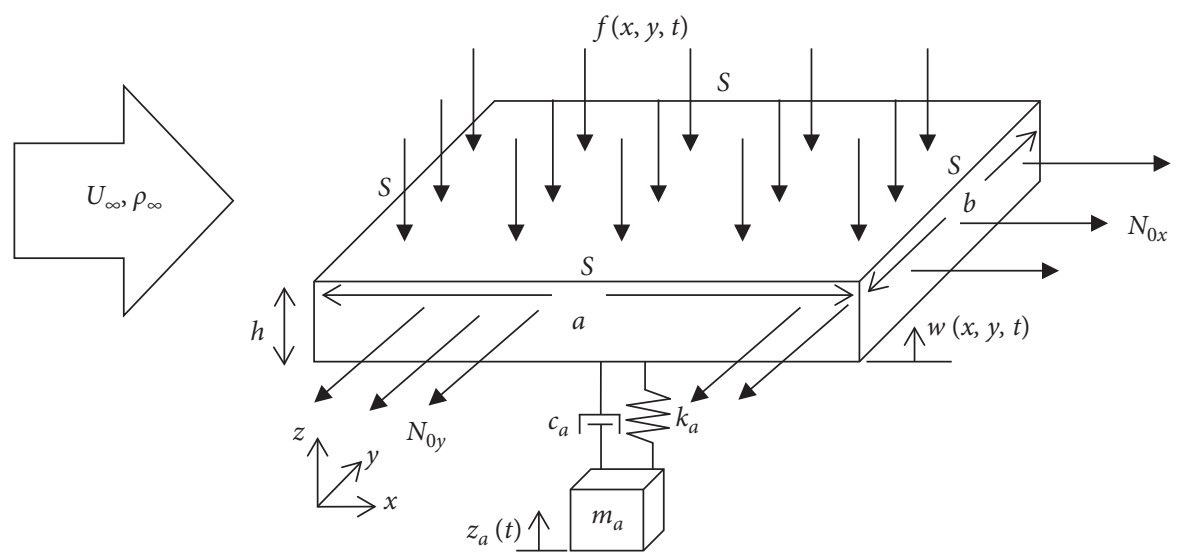

Figure 2: Schematic representation of a suppressed plate.

where $z_{a}, \dot{z}_{a}$, and $\ddot{z}_{a}$ are the displacement, the velocity, and the acceleration of the TMD, respectively. Moreover, $w((a / 2),(b / 2), t)$ and $\dot{w}((a / 2),(b / 2), t)$ are the deflection time history and the velocity time history of the midpoint of the plate, respectively. $\delta(\cdot)$ is also a representation of the Dirac delta function. Considering $w$ of the form $w(x, y, t)=$ $\sin (\pi x / a) \sin (\pi y / b) q(t)$ and multiplying both sides of equation (14) by $\sin (\pi x / a) \sin (\pi y / b)$ and then performing integral from 0 to $a$ for $x$ and from 0 to $b$ for $y$ lead to a set of coupled nonlinear differential equations for $q(t)$ and $z_{a}(t)$ in the form

$$
\left\{M \ddot{q}+C \dot{q}+K q+N q^{3}=f(t)+\frac{4}{a b} k_{a}\left(z_{a}-q\right)+\frac{4}{a b} c_{a}\left(\dot{z}_{a}-\dot{q}\right), m_{a} \ddot{z}_{a}+k_{a}\left(z_{a}-q\right)+c_{a}\left(\dot{z}_{a}-\dot{q}\right)=0\right.
$$

All the parameters used in equation (15) are previously introduced. The Laplace transform technique in conjunction with Adomian Decomposition Method (ADM) can be utilized to obtain the solution of equation (15). By taking Laplace transform from both sides of equation (15), one has

$$
\left\{\begin{array}{l}
M s^{2} Q(s)+C s Q(S)+K Q(s)+N \ell\left\{q^{3}\right\}=F(s)+\frac{4}{a b} k_{a}\left(Z_{a}(s)-Q(S)\right)+\frac{4}{a b} c_{a}\left(s Z_{a}(s)-s Q(S)\right), \\
m_{a} s^{2} Z(S)+k_{a}\left(Z_{a}(s)-Q(S)\right)+c_{a}\left(s Z_{a}(s)-s Q(S)\right)=0
\end{array}\right.
$$

where $\quad Q(S)=\ell\{q(t)\}=\int_{0}^{+\infty} q(t) e^{-s t} \mathrm{~d} t \quad$ and $Z_{a}(s)=\ell\left\{z_{a}(t)\right\}=\int_{0}^{+\infty} z_{a}(t) e^{-s t} \mathrm{~d} t$. One can now employ Adomian Decomposition Method to write the solution of equation (15) as [27]

$$
\left\{\begin{array}{l}
q(t)=\sum_{k=0}^{\infty} q_{k}(t), \\
z_{a}(t)=\sum_{k=0}^{\infty} z_{k}(t) .
\end{array}\right.
$$

Moreover, according to Adomian Decomposition Method, one can propose the nonlinear term of $q^{3}$ as follows [27]:

$$
\widehat{n}_{q}=q^{3}=\sum_{k=1}^{\infty} a_{k},
$$

where $a_{k}$ are Adomian polynomials. By substituting equations (17) and (18) into (16), one has 


$$
\left\{\begin{array}{l}
M s^{2} \sum_{k=0}^{\infty} Q_{k}(s)+C s \sum_{k=0}^{\infty} Q_{k}(s)+K \sum_{k=0}^{\infty} Q_{k}(s)+N\left\{\sum_{k=1}^{\infty} A_{k}(s)\right\}=F(s)+\frac{4}{a b} k_{a}\left(\sum_{k=0}^{\infty} Z_{k}(s)-\sum_{k=0}^{\infty} Q_{k}(s)\right)+\frac{4}{a b} c_{a} s\left(\sum_{k=0}^{\infty} Z_{k}(s)-\sum_{k=0}^{\infty} Q_{k}(s)\right) \\
m_{a} s^{2} \sum_{k=0}^{\infty} Z_{k}(s)+k_{a}\left(\sum_{k=0}^{\infty} Z_{k}(s)-\sum_{k=0}^{\infty} Q_{k}(s)\right)+c_{a} s\left(\sum_{k=0}^{\infty} Z_{k}(s)-\sum_{k=0}^{\infty} Q_{k}(s)\right)=0
\end{array}\right.
$$

Solving linear algebraic equation of (19), one can obtain $Q(s)$ as

$$
Q(s)=\sum_{k=0}^{\infty} Q_{k}(s)=\frac{\left(m_{a} s^{2}+c_{a} s+k_{a}\right)\left(F(s)-N\left(\sum_{k=1}^{\infty} a_{k}\right)\right.}{\left(\tilde{m} s^{2}+\widehat{c} s+\widehat{k}\right)\left(m_{a} s^{2}+c_{a} s+k_{a}\right)-(4 / a b)\left(k_{a}+c_{a} s\right)^{2}},
$$

and also one can obtain $Z(s)$ as

$$
Z_{a}(s)=\sum_{k=0}^{\infty} Z_{k}(s)=\frac{\left(k_{a}+c_{a} s\right)\left(F(s)-N\left(\sum_{k=1}^{\infty} a_{k}\right)\right.}{\left(\bar{m} s^{2}+\tilde{c} s+\hat{k}\right)\left(m_{a} s^{2}+c_{a} s+k_{a}\right)-(4 / a b)\left(k_{a}+c_{a} s\right)^{2}}
$$

where $\quad \widehat{m}=M, \widehat{c}=c+(4 / a b) c_{a}$, and $\widehat{k}=k+(4 / a b) k_{a}$. Now, one can generate the recursive relation of $a_{k}=q_{k-1}^{3}$ $(k=1,2,3, \ldots)$ and obtain

$$
\begin{aligned}
& \left\{\begin{array}{l}
Q_{0}(s)=\frac{\left(m_{a} s^{2}+c_{a} s+k_{a}\right) F(s)}{\left(\widehat{m} s^{2}+\widehat{c} s+\widehat{k}\right)\left(m_{a} s^{2}+c_{a} s+k_{a}\right)-(4 / a b)\left(k_{a}+c_{a} s\right)^{2}} \\
Q_{1}(s)=\frac{-N Q_{0}^{3}}{\widehat{m} s^{2}+\widehat{c} s+\widehat{k}}, \\
Q_{2}(s)=\frac{-N Q_{1}^{3}}{\bar{m} s^{2}+\widehat{c} s+\widehat{k}} \\
\ldots,
\end{array}\right. \\
& \left\{\begin{array}{l}
Z_{0}(s)=\frac{\left(k_{a}+c_{a} s\right) F(s)}{\left(\widehat{m} s^{2}+\hat{c} s+\hat{k}\right)\left(m_{a} s^{2}+c_{a} s+k_{a}\right)-(4 / a b)\left(k_{a}+c_{a} s\right)^{2}}, \\
Z_{1}(s)=\frac{-N Z_{0}^{3}}{\widehat{m} s^{2}+\hat{c} s+\widehat{k}} \\
Z_{2}(s)=\frac{-N Z_{1}^{3}}{\widehat{m} s^{2}+\widehat{c} s+\widehat{k}},
\end{array}\right.
\end{aligned}
$$


One may use Routh's stability criterion [28] to determine the stability threshold of the solutions of equations (22) and (23). Based on Routh's stability criterion, the solutions of equations (22) and (23) are stable if the following hold:

$$
\begin{aligned}
& \widehat{m} k_{a}+\widehat{k} m_{a}+\widehat{c} c_{a}>\frac{4}{a b} c_{a}^{2}, \\
& \frac{\widehat{c}}{c_{a}}+\frac{\hat{k}}{k_{a}}>\frac{8}{a b}, \\
& \widehat{k}>\frac{4}{a b} k_{a} .
\end{aligned}
$$

Numerical methods such as Durbin's numerical Laplace inversion scheme can be utilized to obtain $q_{0}, q_{1}, q_{2}, \ldots, z_{0}$, $z_{1}, z_{2}, \ldots$ in the following forms [16]:

$$
\begin{gathered}
q_{k}(t)=\frac{2 e^{\lambda t}}{\tau} \times\left\{\frac{1}{2} \operatorname{Re}\left(Q_{k}(\lambda)\right)+\sum_{m=1}^{M} \operatorname{Re}\left(Q_{k}\left(\lambda+j \frac{2 m \pi}{\tau}\right)\right) \cos \left(\frac{2 m \pi}{\tau} t\right)-\operatorname{Im}\left(Q_{k}\left(\lambda+j \frac{2 m \pi}{\tau}\right)\right) \sin \left(\frac{2 m \pi}{\tau} t\right), \quad k=0,1,2, \ldots,\right. \\
z_{k}(t)=\frac{2 e^{\lambda t}}{\tau} \times\left\{\frac{1}{2} \operatorname{Re}\left(Z_{k}(\lambda)\right)+\sum_{m=1}^{M} \operatorname{Re}\left(Z\left(\lambda+j \frac{2 m \pi}{\tau}\right)\right) \cos \left(\frac{2 m \pi}{\tau} t\right)-\operatorname{Im}\left(Z\left(\lambda+j \frac{2 m \pi}{\tau}\right)\right) \sin \left(\frac{2 m \pi}{\tau} t\right), \quad k=0,1,2, \ldots,\right.
\end{gathered}
$$

where $\lambda$ is an arbitrary real number greater than all the real parts of the singularities of $Q_{k}(\lambda)$ and $Z_{k}(\lambda)$. Moreover, $Q_{k}(\lambda)$ and $Z_{k}(\lambda)$ should be defined in the interval $[0,2 \tau]$. For sufficient accuracy, the suggested value of " $\lambda t$ " is given with the appropriate sign by [29]

$$
\lambda t=-2 \ln \left\{N_{\tau}\right\}
$$

where $N \tau$ is the number of points in the time signal.

2.3. Calculation of the Acoustic Pressure. The vibrating plate of Figure 1 in an infinite baffle together with the observation point for calculating the acoustic pressure has been schematically depicted in Figure 3.

The acoustic pressure $P_{a c}\left(x_{0}, y_{0}, z_{0}, t\right)$ at the observation point $\Pi\left(x_{0}, y_{0}, z_{0}\right)$ at time $t$ caused by vibration of the plate is given by [5]

$$
P_{a c}\left(x_{0}, y_{0}, z_{0}, t\right)=\frac{\rho_{s}}{2 \pi} \int_{0}^{b} \int_{0}^{a} \ddot{w}\left(x, y, t-\frac{R}{c_{0}}\right) \frac{1}{R} \mathrm{~d} x \mathrm{~d} y,
$$

where $\ddot{w}(x, y, t)$ is the acceleration time history of the plate at $(x, y), c_{0}$ is the wave velocity of the acoustic medium, $\rho_{a m}$ is the mass density of the acoustic medium, and $R$ is the distance between the observation point $\Pi$ and the infinitesimal element at $(x, y)$ on the plate surface. By dividing the sides $a$ and $b$ of the plate into $N_{x}$ and $N_{y}$ equal segments of lengths $\Delta x$ and $\Delta y$, respectively, and using the standard trapezoidal rule [30], the acoustic pressure can be expressed as

$$
\begin{gathered}
P_{a c}\left(x_{0}, y_{0}, z_{0}, t\right)=\frac{\rho_{a m} \Delta x \Delta y}{8 \pi} \sum_{k=0}^{m-1} \sum_{l=0}^{n-1} \frac{\ddot{w}\left(r \Delta x, s \Delta y, t-\left(R_{r, s} / c_{0}\right)\right)}{R_{r, s}}+\frac{\ddot{w}\left((r+1) \Delta x, s \Delta y, t-\left(R_{r+1, s} / c_{0}\right)\right)}{R_{r+1, s}} \\
+\frac{\ddot{w}\left(r \Delta x,(s+1) \Delta y, t-\left(R_{r, s+1} / c_{0}\right)\right)}{R_{r, s+1}}+\frac{\ddot{w}\left((r+1) \Delta x,(s+1) \Delta y, t-\left(R_{r+1, s+1} / c_{0}\right)\right)}{R_{r+1, s+1}} . \\
\text { Here, } R_{r, s} \text { is the distance between the observation point } \Pi \quad R_{r, s}=\sqrt{\left(x_{0}-r \Delta x\right)^{2}+\left(y_{0}-s \Delta y\right)^{2}+z_{0}^{2}},
\end{gathered}
$$
and the element at $(r \Delta x, s \Delta y)$ on the surface of the plate. Furthermore, one can define and $\ddot{q}(t)$ can be acquired from equation (25) and then replaced in equation (29) in order to determine the acoustic pressure as 


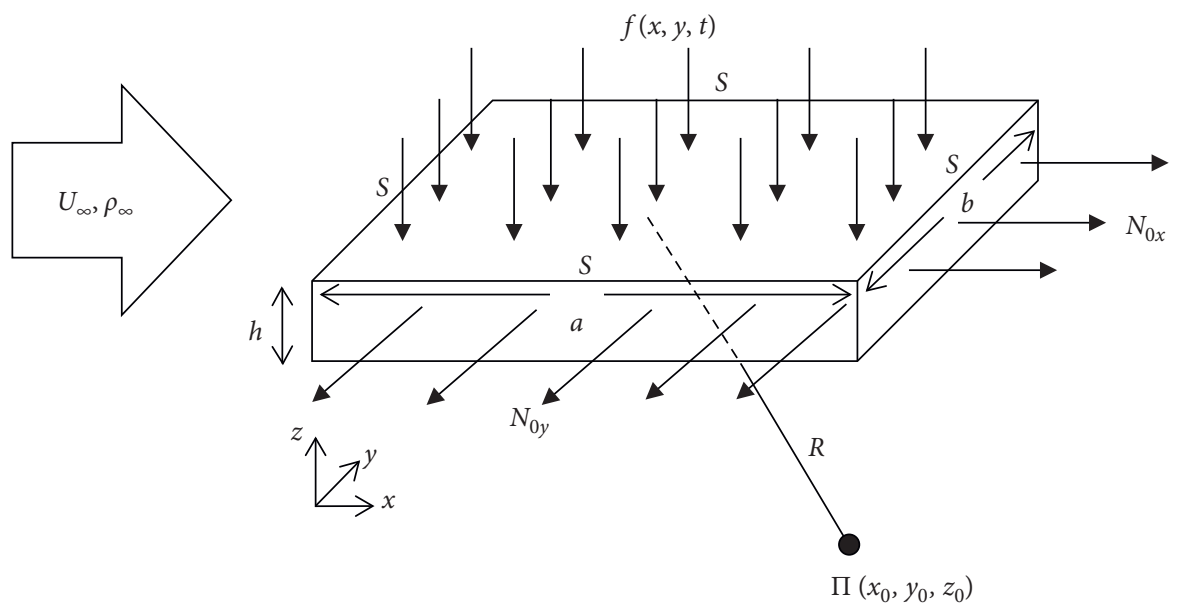

FIGURE 3: Observation point in which the acoustic pressure is evaluated.

$$
\begin{aligned}
P_{a c}\left(x_{0}, y_{0}, z_{0}, t\right)= & \frac{\rho_{a m} \Delta x \Delta y}{8 \pi} \sum_{k=0}^{1} \sum_{l=0}^{1} \sum_{r=0}^{m-1} \sum_{s=0}^{n-1}\left(\frac{\sin ((\pi(r+k) \Delta x) / a) \sin ((\pi(s+l) \Delta y) / b)}{R_{r+k, s+l}}\right. \\
& \times\left\{\ddot{\alpha}_{r+k, s+l} \cos \varphi_{r+k, s+l}-2 \dot{\alpha}_{r+k, s+l} \dot{\varphi}_{r+k, s+l} \sin \varphi_{r+k, s+l}-\alpha_{r+k, s+l} \ddot{\varphi}_{r+k, s+l} \sin \varphi_{r+k, s+l}-\alpha_{r+k, s+l} \dot{\varphi}_{r+k, s+l}^{2} \cos \varphi_{r+k, s+l}\right. \\
& +\frac{3 \varepsilon N}{32 K}\left(\left(2 \alpha_{r+k, s+l} \dot{\alpha}_{r+k, s+l}^{2}+\alpha_{r+k, s+l}^{2} \ddot{\alpha}_{r+k, s+l}-\alpha_{r+k, s+l}^{3} \dot{\varphi}_{r+k, s+l}^{2}\right) \cos \varphi_{r+k, s+l}\right. \\
& \left.-\left(6 \alpha_{r+k, s+l}^{2} \dot{\alpha}_{r+k, s+l} \dot{\varphi}_{r+k, s+l}+\alpha_{r+k, s+l}^{3} \ddot{\varphi}_{r+k, s+l}\right) \sin \varphi_{r+k, s+l}\right) \\
& \left.\left.-\varepsilon \frac{M f_{0}}{K-M \psi^{2}} \psi^{2} \cos \Theta_{r+k, s+l}\right\}\right),
\end{aligned}
$$

where $\varphi=\sqrt{(K / M)} t+\beta(t)$ and $\Theta(t)=\psi t$. Moreover, subscripts $r+k$ and $s+l$ describe the parameter evaluation at time $t-\left(R_{r+k, s+l} / c_{0}\right)$.

\section{Results and Discussion}

In this section, a case study is organized, and the results are reported. The properties of the rectangular elastic plate chosen for this study are the length of $a=2 \mathrm{~m}$, the width of $b=1 \mathrm{~m}$, and the thickness of $h=1.5 \mathrm{~mm}$. Two types of material are selected for the plate: the first type is aluminum with Young's modulus of $E=69 \mathrm{GPa}$, mass density of $\rho=2700 \mathrm{~kg} / \mathrm{m}^{3}$, and Poisson's ratio of $v=0.32$ and the second type is steel with Young's modulus of $E=207 \mathrm{GPa}$, mass density of $\rho=7800 \mathrm{~kg} / \mathrm{m}^{3}$, and Poisson's ratio of $v=0.3$. The viscous damping is also considered as $c=0.01 \mathrm{~N} \mathrm{~s} / \mathrm{m}$ for two cases. The time history response of the midpoint of the plate is illustrated in Figure 4 . These responses have been obtained using two methods of the Multiple Time Scale and Runge-Kutta 4th-order numerical integration method. There is an acceptable coincidence between results acquired from two approaches and one can obtain the response of the equation (15) using Runge-Kutta method.
Figure 5 illustrates the acoustic pressure against the airflow speed for two different materials. It can be found that increasing the speed of the flow causes sound pressure to be reduced. Moreover, the acoustic pressure is dominantly increasing for the speeds in the vicinity of a critical speed in which the resonance phenomenon occurs in the plate (i.e., $\sqrt{K / M} \approx \psi$ ). It is worth noting that, in the practical range of HST speeds $\left(U_{\infty}>250 \mathrm{~km} / \mathrm{h}\right)$, the acoustic pressure generated from the steel plate is significantly larger than that generated from the aluminum plate.

The acoustic pressure versus the aspect ratio (i.e., $a / b$ ) of the plate has been plotted in Figure 6. It is observed that the sound pressure decreases as the aspect ratio increases. It is also found that, in the large aspect ratios (say $a / b>4$ ), the acoustic pressure is independent of the plate material.

In Figure 7, the acoustic pressure has been plotted versus the plate thickness. At first, it can be found from the figure that the thick plates generate lower acoustic pressure than that of the thin plates. This phenomenon occurs because of high rigidity in the thick plates. Plates with higher rigidities have small deformations in the vertical directions. Accordingly, the acoustic pressure generated from these plates has lower levels. Moreover, in the plates with the moderate 


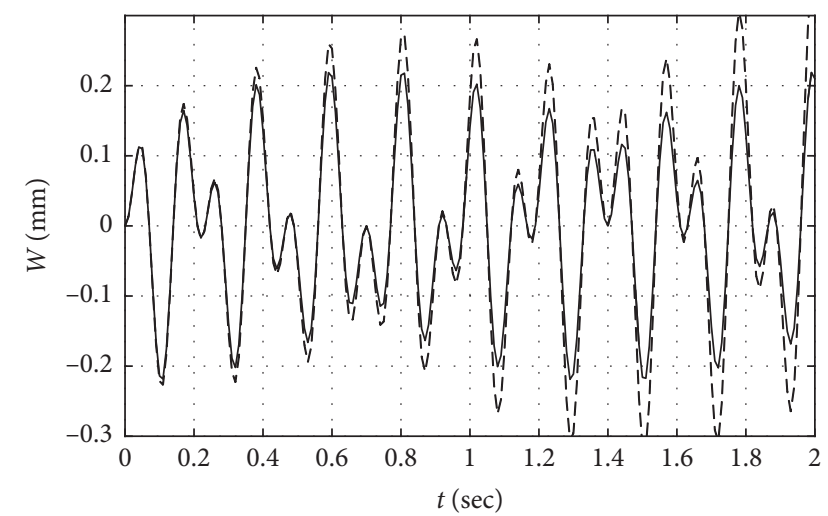

- Multiple Time Scale

- - ODE45

Figure 4: The time response of the midpoint of the plate using Multiple Time Scale Method and numerical solutions.

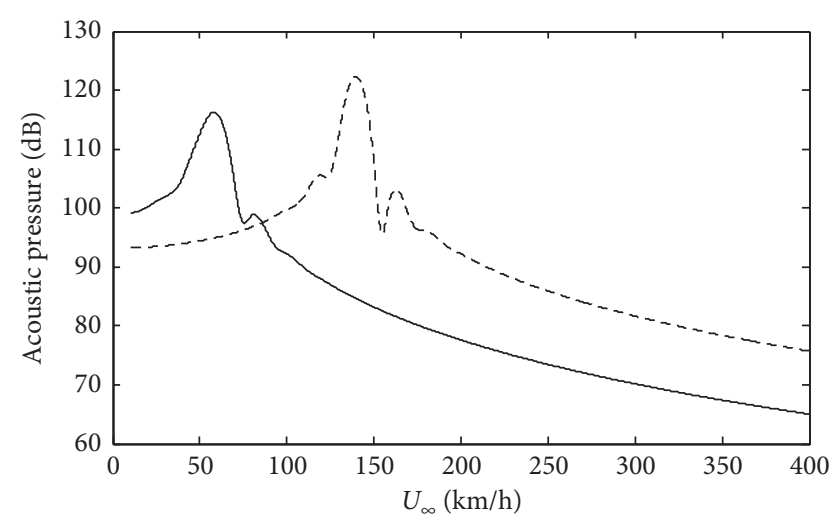

- Aluminum

- - - Steel

FIGURE 5: The acoustic pressure against the flow speed for different plate materials.

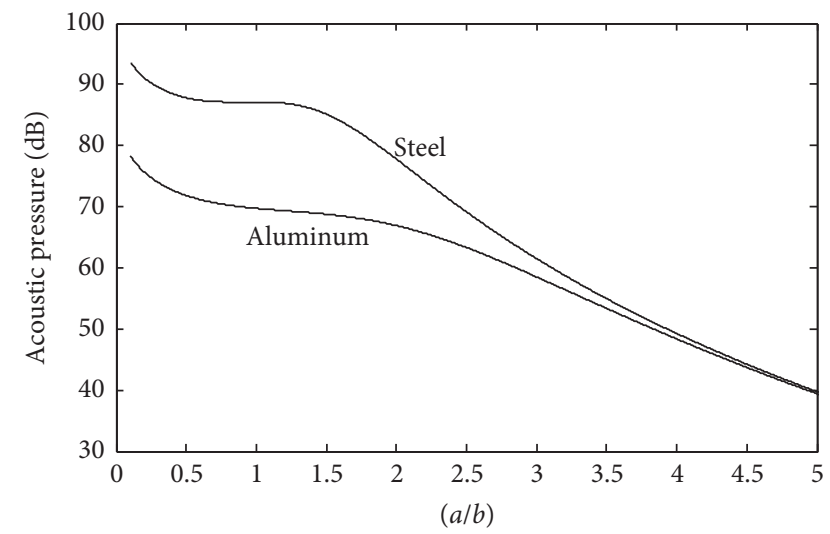

FIGURE 6: The acoustic pressure versus the aspect ratio of the plate for different plate materials.

thickness (say $5 \mathrm{~mm}$ in our study), the sound pressure is independent of the plate material. This is from the amazing outcomes of the present study.

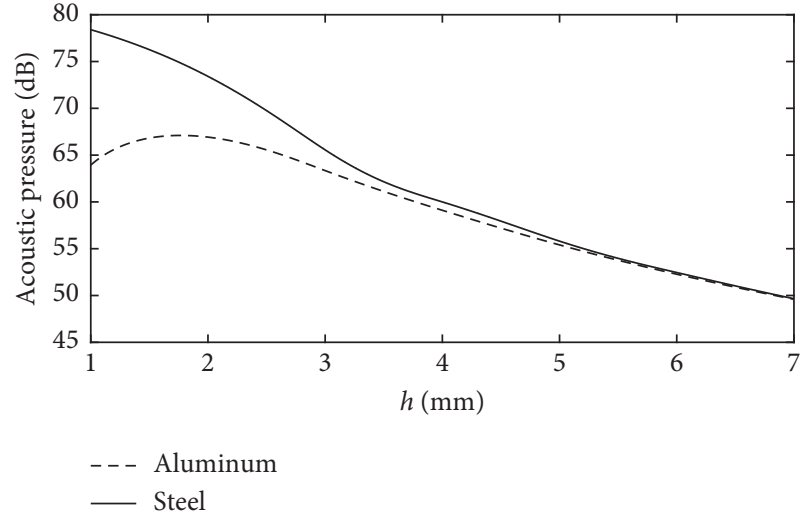

FIGURE 7: The acoustic pressure versus plate thickness for different plate materials.

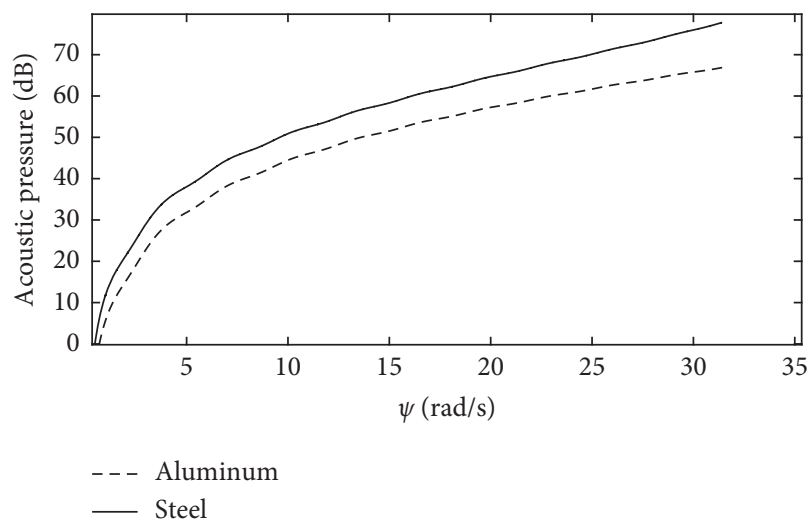

FIgURE 8: The influence of the forcing frequency on the acoustic pressure.

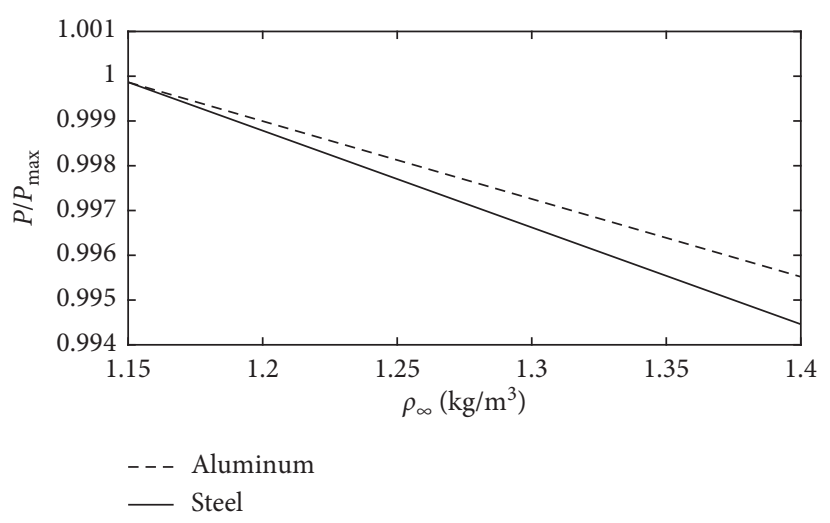

FIGURE 9: Influence of the airflow density on the acoustic pressure.

Figure 8 represents the acoustic pressure generated from the plate versus the frequency of the external force. The figure shows that the forces with higher frequencies can cause larger acoustic pressures. Increasing the forcing frequencies means approaching the natural frequencies of the plates and occurrence of the resonance phenomenon. It is 


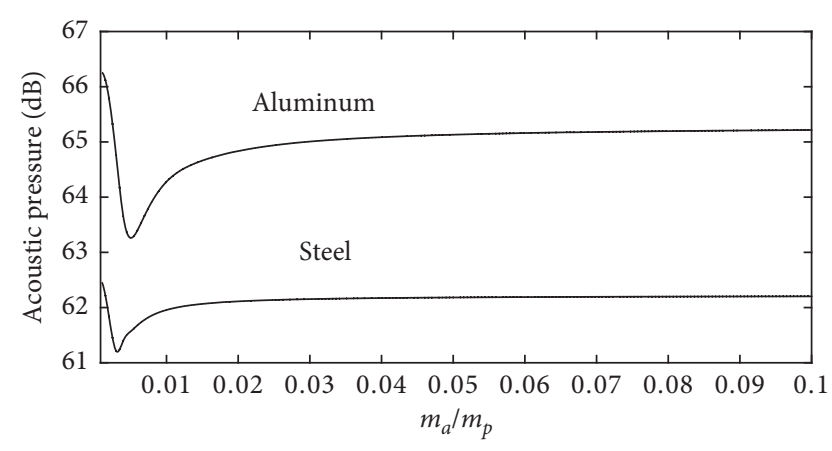

FIGURE 10: The acoustic pressure versus the mass ratio.

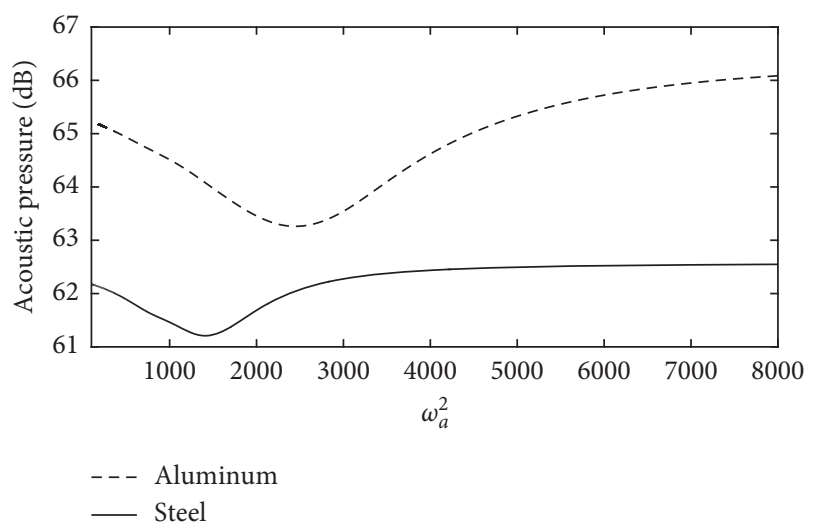

Figure 11: The acoustic pressure versus the natural frequency of the TMD.

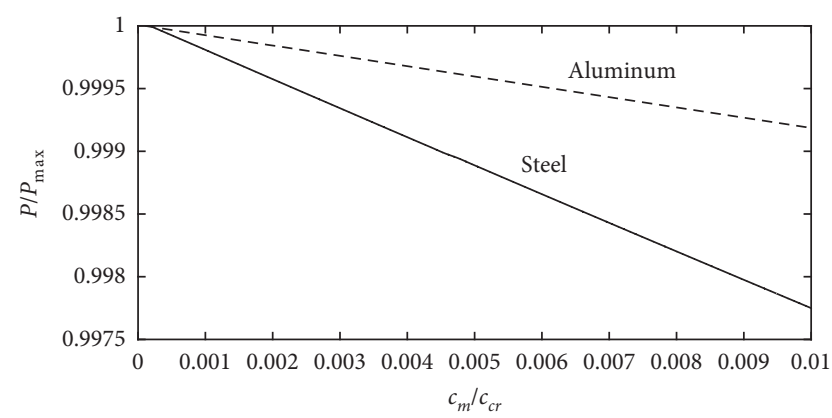

FIGURE 12: The acoustic pressure versus the damping ratio of the TMD.

also known that the difference between the sound pressures generated from the aluminum and the steel plates becomes larger with increasing the frequency of the external force. This difference is because of the different natural frequencies of aluminum and steel plates. It is found from this figure that the selected frequencies are near to the natural frequency of the studying steel plate.

The influence of the airflow density on the acoustic pressure has been shown in Figure 9. It is clear that when the density of the airflow increases (i.e., when the temperature of the airflow decreases), the acoustic pressure is reduced, but the airflow density does not play an important role in the

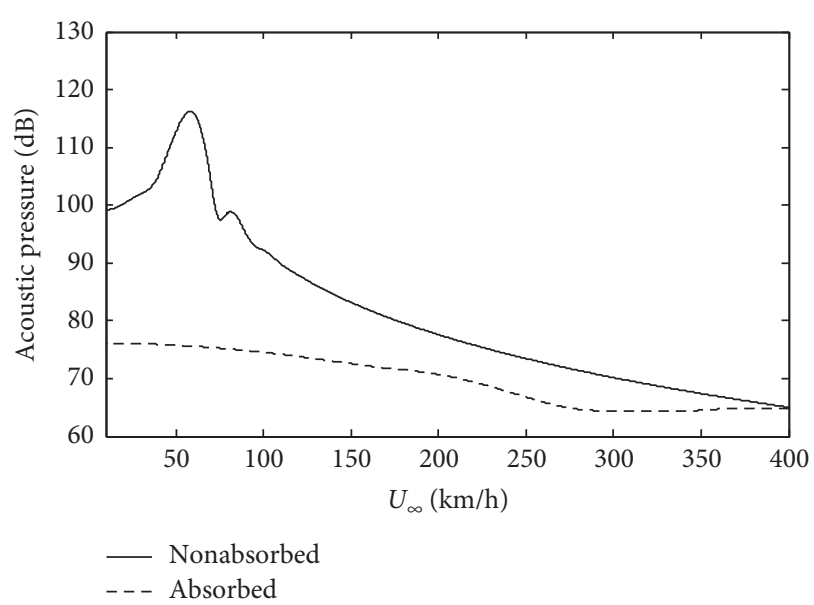

FIgURE 13: The acoustic pressure versus the speed for two cases: with and without TMD system.

sound pressure generation. For example, if the density of the passing flow decreases about 20\% (when the temperature decreases from $35^{\circ} \mathrm{C}$ to $-25^{\circ} \mathrm{C}$ ), the sound pressure received from the plate decreases about $0.5 \%$ (i.e., $P_{\max }$ is reduced to $\left.0.995 P_{\max }\right)$. According to equations (3) and (4), the airflows with higher mass densities passing through the vibrating plate increase the stiffness of the plate and decrease the vibrational amplitudes. Therefore, this can cause the acoustic pressure to take lower levels. As the density of the airflow cannot extremely change in the ambient temperature, the sound pressure changes lightly versus the mass density of the airflow in this condition. It is also discovered from Figure 9 that the steel plate is more sensitive to the airflow density rather than the aluminum plate.

Figure 10 compares the sound pressure generated against the mass ratio (i.e., $\left.\left(m_{a} / m_{p}\right)\right)$ for the aluminum and the steel alloys. It is observed that there is an optimal choice for the mass ratio in which the acoustic pressure takes its minimum value. This optimal ratio depends on the plate material $(\sim 0.005$ for the aluminum and $\sim 0.003$ for the steel alloys). An important result can be obtained from a comparison of Figures 5 through 8 with Figure 10. It says that using the TMD system causes the acoustic pressure received from the aluminum plates to take larger values compared to that of the steel plates, although this pattern is completely the opposite without using the TMD system.

Figure 11 investigates the sound pressure against the natural frequency of the TMD (i.e., $\left.\omega_{a}^{2}=\left(k_{a} / m_{a}\right)\right)$. It is seen that, for an optimum value of $\left(k_{a} / m_{a}\right)$, the TMD has a good efficiency in the reduction of the sound propagation. This optimum value differs from the aluminum plate to the steel plate. The influence of the damping ratio (i.e., $\left.\left(c_{a} / c_{c r}\right)\right)$ on the acoustic pressure has been demonstrated in Figure 12. In this graph, the general behavior is the same as that shown in Figure 9.

Using a TMD in the midpoint of the plate can noticeably decrease the sound pressure radiated from the aluminum plate. This pattern is observed against the airflow speed in Figure 13. It is clear from the figure that the efficiency of the TMD can be reduced in high-speed regimes. 


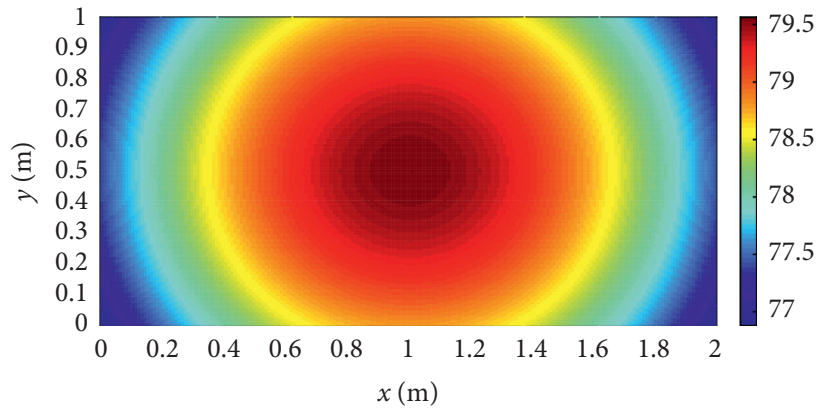

(a)

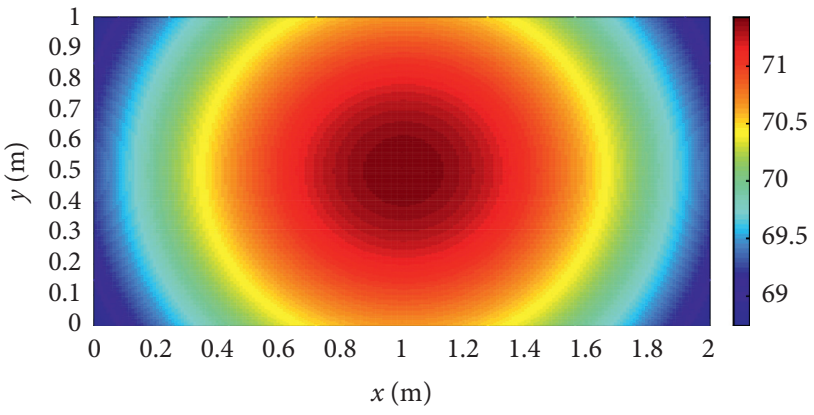

(b)

FIGURE 14: The noise contour maps (a) before and (b) after using the TMD system.

The noise contour maps around the vibrating plate are shown in Figure 14. The contours have been created at a distance of 1 meter away from the surface of the aluminum plate (i.e., $R=1 \mathrm{~m}$ ) before and after using the TMD system. It is obvious from the contour maps that the maximum value of the acoustic pressure is received along the midpoint of the plate.

Figure 14 demonstrates that using the TMD system can remarkably reduce the noise level (from $\sim 80 \mathrm{~dB}$ to $\sim 72 \mathrm{~dB}$ in this study).

\section{Conclusions}

The acoustic pressure generated from the plates with geometric nonlinearity subjected to subsonic airflow and external excitation was studied in this paper. At first, the vibrations of the plate were analyzed based on von-Kármán's assumptions. The airflow was considered as the potential flow and the pressure distribution was obtained. Adding a tuned mass damper (TMD) system to the surface of the plate led to absorption in the vibrations of the plate. The solution of the plate-absorber dynamical system was obtained analytically employing the Laplace transform technique and Adomian Decomposition Method. Rayleigh integral technique and Boundary Element Method were used to determine the acoustic pressure distribution around the plate. In a parametric study, the effects of the airflow speed, the aspect ratio, and TMD properties on the sound pressure were investigated. The following are the main outcomes of this study:

(i) Increasing the speed of the airflow passing through the plate leads to decreasing the acoustic pressure received from the plate.

(ii) The aluminum plates have lower values of acoustic pressure than the steel plates in the absence of TMD. This pattern is completely different in the presence of TMDs.

(iii) Plates with higher values of aspect ratios generate low values of acoustic pressure. Increasing the aspect ratio also makes the acoustic pressure independent from the plate's material.

(iv) Thick plates generate lower acoustic pressure compared to thin plates. Additionally, in the thick plates (when the plate thickness is more than $5 \mathrm{~mm}$ in our study), the acoustic pressure is independent of the plate material.

(v) There are optimum values for the mass ratio and the natural frequency of the TMD system in which the acoustic pressure takes its minimum value.

\section{Data Availability}

The data that support the findings of this study are available from the corresponding author upon reasonable request.

\section{Conflicts of Interest}

The authors declare that they have no conflicts of interest.

\section{References}

[1] D. Younesian and H. Norouzi, "Frequency analysis of the nonlinear viscoelastic plates subjected to subsonic flow and external loads," Thin-Walled Structures, vol. 92, pp. 65-75, 2015.

[2] S. Dastjerdi, B. Akgöz, and Ö. Civalek, "On the effect of viscoelasticity on behavior of gyroscopes," International Journal of Engineering Science, vol. 149, Article ID 103236, 2020.

[3] S. V. Sorokin, "Vibrations of and sound radiation from sandwich plates in heavy fluid loading conditions," Composite Structures, vol. 48, no. 4, pp. 219-230, 2000.

[4] F. Chong, Y. Y. Lee, and C. F. Ng, "Vibration and sound radiation from plates excited by piezoelectric actuators," Building Acoustics, vol. 8, no. 3, pp. 179-198, 2001.

[5] F. Au and M. Wang, "Sound radiation from forced vibration of rectangular orthotropic plates under moving loads," Journal of Sound and Vibration, vol. 281, no. 3-5, pp. 10571075, 2005.

[6] K. Akamatsu, T. Yamaguchi, and J. Kanazawa, "Sound radiation from finite plates excited by a space-time stream of random impulses," The Journal of the Acoustical Society of America, vol. 120, no. 5, p. 3345, 2006.

[7] H. Denli and J. Sun, "Structural-acoustic optimization of sandwich structures with cellular cores for minimum sound radiation," Journal of Sound and Vibration, vol. 301, no. 1-2, pp. 93-105, 2007.

[8] P. Jeyaraj, C. Padmanabhan, and N. Ganesan, "Vibration and acoustic response of an isotropic plate in a thermal 
environment," Journal of Vibration and Acoustics, vol. 130, no. 5, 2008.

[9] J. Da Rocha, A. Suleman, and F. Lau, "Prediction of flowinduced noise in transport vehicles: development and validation of a coupled structural-acoustic analytical framework," Canadian Acoustics, vol. 37, no. 4, pp. 13-29, 2009.

[10] F. X. Xin and T. J. Lu, "Analytical modeling of sound transmission across finite aeroelastic panels in convected fluids," The Journal of the Acoustical Society of America, vol. 128, no. 3, pp. 1097-1107, 2010.

[11] M. Smith and E. L. Iglesias, "Vibration and noise radiation from a panel excited by a turbulent flow," Acoustics, vol. 2012, 2012.

[12] J. Rocha, "Sound radiation and vibration of composite panels excited by turbulent flow: analytical prediction and analysis," Shock and Vibration, vol. 2014, Article ID 316481, 2014.

[13] M. M. Sucheendran, D. J. Bodony, and P. H. Geubelle, "Coupled structural-acoustic response of a duct-mounted elastic plate with grazing flow," AIAA Journal, vol. 52, no. 1, pp. 178-194, 2014.

[14] B. Akgöz and Ö. Civalek, "A microstructure-dependent sinusoidal plate model based on the strain gradient elasticity theory," Acta Mechanica, vol. 226, no. 7, pp. 2277-2294, 2015.

[15] T. Yang, W. Zheng, Q. Huang, and S. Li, "Sound radiation of functionally graded materials plates in thermal environment," Composite Structures, vol. 144, pp. 165-176, 2016.

[16] R. Shakeri and D. Younesian, "Broad-band noise mitigation in vibrating annular plates by dynamic absorbers," International Journal of Structural Stability and Dynamics, vol. 16, no. 6, Article ID 1550014, 2016.

[17] K. Daneshjou, R. Talebitooti, and A. Tarkashvand, “An exact solution of three-dimensional elasticity for sound transmission loss through FG cylinder in presence of subsonic external flow," International Journal of Mechanical Sciences, vol. 120, pp. 105-119, 2017.

[18] N. Chandra, K. V. Nagendra Gopal, and S. Raja, "Vibroacoustic response of sandwich plates with functionally graded core," Acta Mechanica, vol. 228, no. 8, pp. 2775-2789, 2017.

[19] Z.-G. Song, T.-Z. Yang, F.-M. Li, E. Carrera, and P. Hagedorn, "A method of panel flutter suppression and elimination for aeroelastic structures in supersonic airflow," Journal of $\mathrm{Vi}$ bration and Acoustics, vol. 140, no. 6, 2018.

[20] W. Zhao, C. Zheng, C. Liu, and H. Chen, "Minimization of sound radiation in fully coupled structural-acoustic systems using FEM-BEM based topology optimization," Structural and Multidisciplinary Optimization, vol. 58, no. 1, pp. 115128, 2018.

[21] A. Chauvin, M. Monteil, S. Bellizzi, R. Côte, P. Herzog, and M. Pachebat, "Acoustic characterization of a nonlinear vibroacoustic absorber at low frequencies and high sound levels," Journal of Sound and Vibration, vol. 416, pp. 244-257, 2018.

[22] K. Zhou, Z. Lin, X. Huang, and H. Hua, "Vibration and sound radiation analysis of temperature-dependent porous functionally graded material plates with general boundary conditions," Applied Acoustics, vol. 154, pp. 236-250, 2019.

[23] M. H. Jalaei and Ö. Civalek, "On dynamic instability of magnetically embedded viscoelastic porous FG nanobeam," International Journal of Engineering Science, vol. 143, pp. 14-32, 2019.

[24] Q. Qin, M. Sheng, M. Wang, C. Wang, and Y. He, "Sound radiation from plates with elastic boundary conditions embedded in an infinite perforated rigid baffle," Journal of Sound and Vibration, vol. 479, Article ID 115361, 2020.
[25] G. Huo, Z. Chen, Y. Jiao, and X. Zhu, "Optimization of dynamic vibration absorbers for suppressing sound radiation of plate structures," Journal of Vibration and Acoustics, vol. 143 , no. $2,2021$.

[26] H. Norouzi and D. Younesian, "Vibro-acoustic numerical analysis for the geometrically nonlinear viscoelastic rectangular plate subjected to subsonic compressible airflow," Applied Acoustics, vol. 174, Article ID 107779, 2021.

[27] E. Esmailzadeh, D. Younesian, and H. Askari, Analytical Methods in Nonlinear Oscillations, Springer, Dordrecht, Netherlands, 2018.

[28] K. Ogata, Modern Control Engineering, Prentice-Hall, Hoboken, NJ, USA, 2010.

[29] J.-F. Blais and A. Ross, "Forward projection of transient sound pressure fields radiated by impacted plates using numerical Laplace transform," The Journal of the Acoustical Society of America, vol. 125, no. 5, pp. 3120-3128, 2009.

[30] S. Schedin, C. Lambourg, and A. Chaigne, "Transient sound fields from impacted plates: comparison between numerical simulations and experiments," Journal of Sound and Vibration, vol. 221, no. 3, pp. 471-490, 1999. 